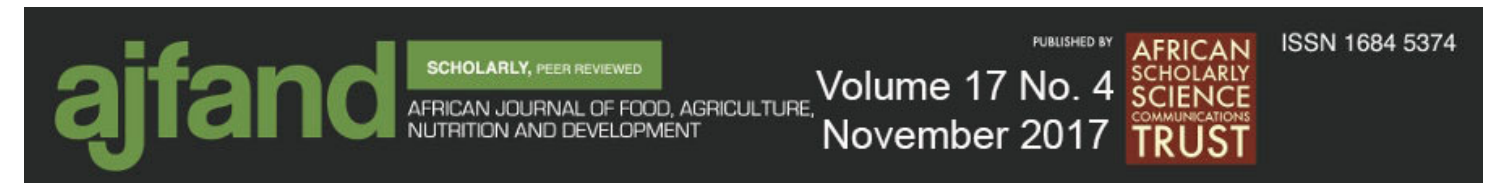

\title{
EVALUATION DES MODES DE PREPARATION ET DE CONSOMMATION DE LA TISANE DE KINKELIBA ET DU CAFE TOUBA CONSOMMES DANS LA VILLE DE DAKAR AU SENEGAL
}

$$
\text { Moussa } \mathbf{N}^{*} \text {, Diallo } \mathrm{A}^{1} \text {, Ndiaye } \mathrm{NF}^{2} \text { and } \mathrm{M} \operatorname{Sadji}^{2}
$$

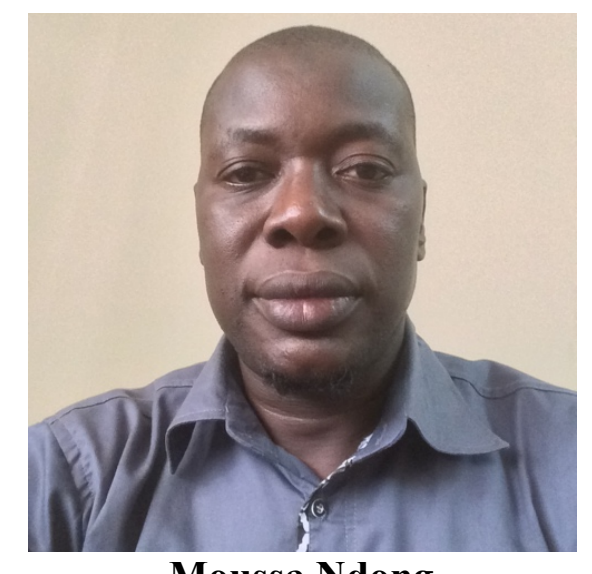

$$
\text { Moussa Ndong }
$$

*Corresponding author email: moussa.ndong@ugb.edu.sn

${ }^{1}$ Section Technologies Agro-Alimentaires, UFR des Sciences Agronomiques, de l'Aquaculture et des Technologies Alimentaires, Université Gaston Berger de SaintLouis, Sénégal

${ }^{2}$ Institut de Technologie Alimentaire, Division Nutrition, Dakar, Sénégal 


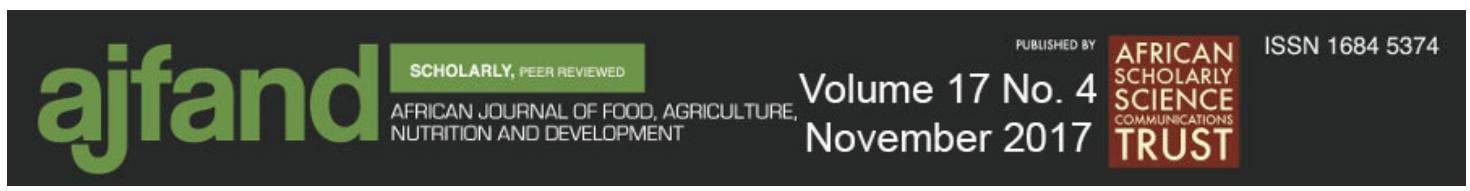

\begin{abstract}
As a consequence of societies' evolution, food habits are changing and resulting in serious issues. Consumers are experiencing new foods and new ways of consuming traditional ones. Café Touba and kinkeliba are important beverages in Senegalese diet. Kinkeliba herbal tea is made of a decoction of dry leaves of Combretum micranthum. Café Touba is made by pouring hot water over roasted, ground coffee (Coffea robusta) and Xylopia aethiopica beans through a filter container. Sugar is added to the beverages, which are generally consumed in breakfast or sold in the streets or in small restaurants called Tangana. Many Senegalese, especially the young, are involved into the business. In this study, a survey was conducted in houses, streets and Tangana where the Café Touba and kinkeliba are prepared, consumed or sold. The objective of the survey was to determine methods of preparation and consumption of two local brews in Dakar. Sixty (60) questionnaires were given to the target people to assess the preparation methods and the amount of drinks consumed. Standard methods of preparation of Café Touba and kinkeliba were defined. From these methods, drinks were prepared and used to determine the tannins contents. The added sugar and energy taken for one portion size were also determined. The results showed differences in preparing methods and drinking habits of Café Touba and kinkeliba. The house-made Café Touba was more concentrated than the one prepared and sold in streets or in Tangana. Total dry leaves used for preparing the house-made kinkeliba or sold in streets or in Tangana were similar. Unlike the kinkeliba, the amount of Café Touba consumed in houses for one serving was higher than that in Tangana or in streets. These drinks could be good sources of tannins which have important therapeutic proprieties. However, Café Touba and kinkeliba drinkers could be exposed to an excessive added sugar consumption. In conclusion, Café Touba and kinkeliba are drinks that are well consumed by Senegalese. Promoting these local brews could improve nutrition and health of consumers. However, the high amount of added sugar in these drinks could increase the risk of chronic diseases.
\end{abstract}

Key words: Café Touba, Kinkeliba, Tannins, Household, Sellers, Cups, Drinks, Breakfast, Sugar 


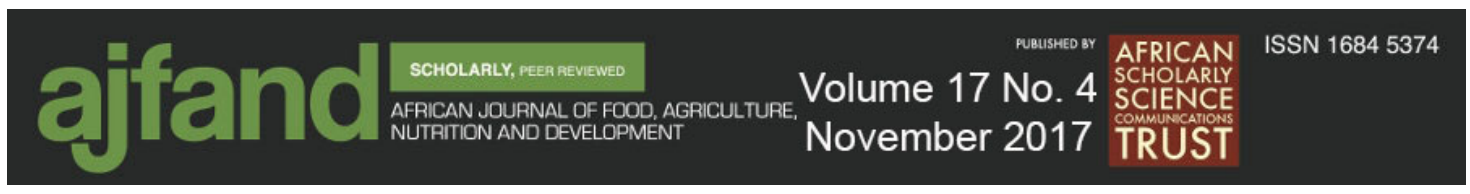

RESUME

Les changements dans les habitudes alimentaires se manifestent par l'introduction de nouveaux aliments ou une nouvelle utilisation des aliments traditionnels. C'est ainsi que le Café Touba et la tisane de kinkéliba sont devenus des aliments incontournables dans le régime alimentaire quotidien de beaucoup de Sénégalais. Le Café Touba est préparé par filtration de la poudre des grains de café (Coffea robusta) et de Xylopia aethiopica. La tisane de kinkéliba est obtenue par décoction de feuilles séchées de Combretum micranthum. Ces boissons sont servies au petit déjeuner ou vendues dans les rues ou dans de petits restaurants appelés communément Tangana. La vente de ces boissons est devenue ainsi une activité économique qui occupe plusieurs jeunes Sénégalais. Dans cette étude, une enquête a été réalisée auprès des ménages et des vendeurs de ces boissons. Un questionnaire a été administré à soixante femmes et à soixante vendeurs pour recueillir des informations sur les modes de préparation et de consommation de ces boissons. Un mode standard de préparation de ces boissons a été défini. Des boissons préparées selon ce dernier ont été analysées pour déterminer les quantités de tanins. Les quantités de sucre ajouté et d'énergies ingérées selon les modes de consommations ont été aussi déterminées. Les résultats ont montré des différences dans les modes de préparation et de consommation. Le Café Touba préparé dans les ménages s'est révélé plus concentré en poudre de café que celui vendu. Pour la tisane de kinkéliba, les quantités de feuilles séchées utilisées sont similaires pour les femmes de ménages et les vendeurs. Contrairement à la tisane de kinkéliba, la quantité de Café Touba consommée dans les ménages par personne est plus grande que celle achetée et consommée. La consommation de ces boissons peut constituer une source de tanins qui ont des effets thérapeutiques importants pour les consommateurs. La promotion de ces deux boissons locales pourrait impacter positivement l'état nutritionnel et sanitaire des consommateurs. Cependant, les quantités de sucre ajoutées pendant la préparation de ces boissons exposent les consommateurs à des apports en sucre supérieurs aux besoins journaliers recommandés. Cette étude a confirmé que Le Café Touba et la tisane de kinkéliba sont des boissons très consommées par les Sénégalais. Leur consommation quotidienne est une source importante de tanins. Cependant, les quantités de sucres ingérées avec ces boissons peuvent augmenter les risques de survenue de maladies chroniques.

Mots clés : Café Touba, Kinkéliba, Tanins, Ménages, Vendeurs, Tasse, Boissons, Petit déjeuner, Sucre 


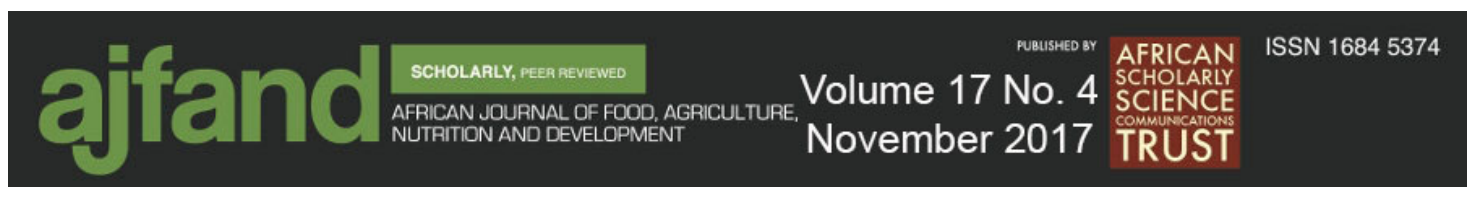

\section{INTRODUCTION}

Le café et la tisane de kinkéliba sont consommés pour leurs propriétés excitantes, tonifiantes et autres effets bénéfiques sur la santé. En effet, le café et la tisane de kinkéliba ont des vertus thérapeutiques pour la prévention de différentes pathologies $[1,2]$. Leurs modes de préparation et de consommation sont différentes selon les sociétés. Au Sénégal, la consommation du café local dénommé " Café Touba » et de la tisane de kinkéliba est largement très répandue dans les ménages, les marchés, les rues et les petits restaurants appelés "Tanganas ». Connues des sénégalais depuis longtemps, ces boissons font partie intégrante de leurs habitudes alimentaires. Le Café Touba est une boisson obtenue par filtration d'un mélange de poudre de grains de café (Coffea robusta) et de gousses d'une épice, Xylopia aethiopica, possédant une forte saveur parfumée et poivrée. Quant à la tisane de kinkéliba, elle est obtenue par décoction de feuilles séchées de Combretum micranthum. Le kinkéliba et le café sont des sources de substances végétales thérapeutiques bénéfiques pour la santé humaine. C'est l'exemple des tanins qui sont très répandus dans le règne végétal [3]. Les tanins jouent un rôle important dans l'absorption des nutriments mais aussi dans la prévention de certaines maladies comme l'hypertension et cancers. Certains des composés fonctionnels des tannins permettent de prévenir plusieurs maladies chroniques non transmissibles [4]. Au Sénégal, même si la vente de ces deux boissons constitue une activité économique qui occupe beaucoup de jeunes sénégalais, leurs modes de préparation et de consommation sont mal connues. C'est dans ce cadre que cette recherche a été initiée pour étudier les modes de préparation et de consommation de ces deux boissons dans les ménages et auprès des vendeurs de Café Touba et de tisane de kinkéliba.

\section{MATERIELS ET METHODES}

L'étude a été conduite dans la périphérie des marchés de la banlieue de Dakar où se concentre la quasi-majorité des groupes socio-ethniques de la population dakaroise.

Le matériel végétal utilisé est composé de : grains de Coffea robusta, gousses de Xylopia aethiopica et de feuilles séchées de Combretum micranthum qui ont tous été obtenus au niveau desdits marchés.

Pour ce qui est de la méthode, l'échantillonnage non probabiliste a été retenu. C'est une méthode pour laquelle le choix des individus n'est pas aléatoire mais raisonné et permet de réduire les coûts de l'étude. Ce choix s'explique par l'aspect cosmopolite de la ville de Dakar (toutes les couches sociales y sont représentées). Les unités statistiques sont composées de femmes de ménages et les vendeurs de Café Touba et de la tisane de kinkéliba.

Pour le processus d'échantillonnage, 60 femmes de ménages et 60 vendeurs ont été choisis au sein des zones d'habitations retenues de façon aléatoire. L'enquête a été menée par des étudiants stagiaires de la Division nutrition de l'Institut de Technologie Alimentaire de Dakar. Ils ont été formés à la méthodologie d'enquête. Les ingrédients ont été pesés par une balance de précision et les volumes mesurés par des éprouvettes. L'échantillonnage s'est fait en choisissant les sujets au sein de la population selon leur disponibilité jusqu'à avoir le nombre nécessaire. Les questionnaires préalablement testés 


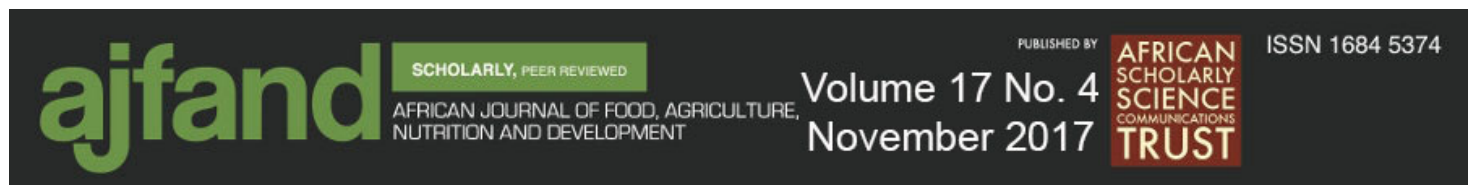

ont été administrés aux enquêtés pour obtenir des informations sur les modes de préparation du Café Touba et de la tisane de kinkéliba ainsi que les quantités consommées. Il s'agit des informations relatives à : (i) la liste des ingrédients ainsi que les quantités utilisées dans la préparation du Café Touba, (ii) la moyenne des produits entrant dans la préparation de la tisane de kinkéliba. Les résultats sont présentés sous forme de moyennes des 60 observations \pm Ecart-type. Les différences entre les groupes sont déterminées par un t-test.

Sur la base de l'enquête, un mode standardisé de préparation du Café Touba et de tisane de kinkéliba a été établi. Les boissons préparées selon ce mode standard ont été utilisées pour déterminer les teneurs en tanins et estimer les apports en sucres ajoutés et en calories induits par la consommation de ces boissons.

La teneur en tanins des produits a été déterminée par la méthode de Folin-Denis modifiée par Seigler et al. [5]. Les composés phénoliques ont été extraits par voie aqueuse avec le réactif de Folin-Denis en solution saturée de carbonate de sodium. La densité optique a été déterminée à $725 \mathrm{~nm}$ par spectrophotomètre UV (ZASCO V-530). La réaction entre le réactif de Folin- Denis et une gamme d'étalonnage a permis d'exprimer les résultats en équivalent acide tannique. Chaque échantillon a été dosé en triplicata.

\section{RESULTATS}

Les données recueillies sur les modes de préparation et de consommation de la tisane de kinkéliba sont présentés dans le Tableau 1.

Ces résultats ont montré que dans les ménages, la tisane de kinkéliba se prépare avec une quantité moyenne de $1,72 \pm 0,90 \mathrm{~L}$ d'eau, $31,2 \pm 19,78 \mathrm{~g}$ de feuilles séchées de kinkéliba et $483,33 \pm 427,47 \mathrm{~g}$ de sucre. En ce qui concerne la consommation, les résultats ont révélé qu'au petit déjeuner, chaque membre du ménage consomme en moyenne une quantité de $181,36 \pm 66,62 \mathrm{ml}$ de ce mélange. Quant aux vendeurs, l'enquête a permis d'obtenir les quantités ci-après : $1,86 \pm 0,55 \mathrm{~L}$ d'eau, $36,46 \pm 22,12 \mathrm{~g}$ de feuilles séchées de Combretum micranthum et $456,66 \pm 307,41 \mathrm{~g}$ de sucre pour la préparation de la tisane de kinkéliba. En moyenne, un volume de $218,26 \pm 82,70 \mathrm{ml}$ est vendu à chaque client pour sa consommation.

Les données recueillies sur les modes de préparation et de consommation du Café Touba sont présentés dans le Tableau 2. Ces résultats ont montré que dans les ménages, le Café Touba se prépare avec une quantité moyenne de $1,53 \pm 0,54 \mathrm{~L}$ d'eau, $107,53 \pm 54,19 \mathrm{~g}$ de poudre de café et $366,66 \pm 99,85 \mathrm{~g}$ de sucre. En ce qui concerne la consommation, les résultats ont révélé qu'au petit déjeuner, chaque membre du ménage consomme en moyenne une quantité de 200,86 $\pm 63,56 \mathrm{ml}$ de cette boisson. Quant aux vendeurs, l'enquête a permis d'obtenir les quantités suivantes : 17,56 $\pm 44,33$ L d'eau, $597 \pm 1255,52$ $\mathrm{g}$ de poudre de Café Touba et $2158,33 \pm 3665,55 \mathrm{~g}$ de sucre. Les résultats ont montré que le mode de préparation de ces boissons est marqué par une forte disparité. En ce qui concerne la consommation, l'étude a montré que la quantité moyenne vendue à chaque client est de $138,53 \pm 37,02 \mathrm{ml}$. 


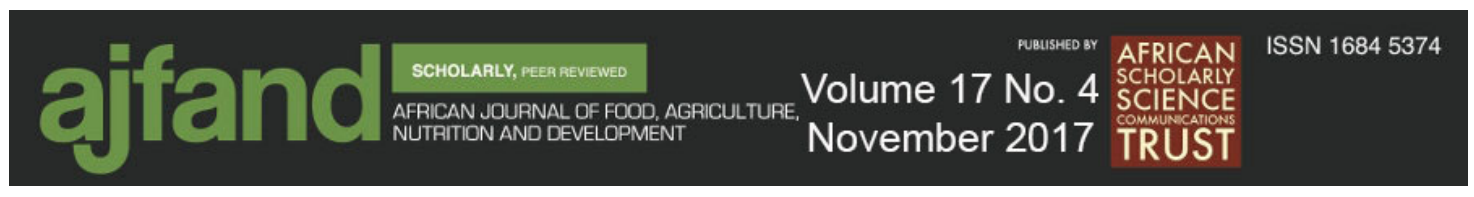

Pour avoir un mode standardisé de préparation de ces boissons, les valeurs de chaque ingrédient utilisé ont été rapportées à un litre d'eau (Tableaux 3 et 4). Les résultats ont montré que dans les ménages, le Café Touba se prépare avec une concentration en poudre deux fois plus élevée que celui préparé par les vendeurs. En effet, pour un litre de Café Touba, les quantités de poudre sont de $70 \mathrm{~g}$ dans les ménages et $34 \mathrm{~g}$ pour les vendeurs. En ce qui concerne le kinkéliba, les quantités utilisées pour la préparation sont similaires entre les vendeurs et les femmes des ménages. En effet, les femmes des ménages et les vendeurs utilisent respectivement $19,5 \mathrm{~g}$ et $18 \mathrm{~g}$ de feuilles séchées pour préparer $1 \mathrm{~L}$ de tisane.

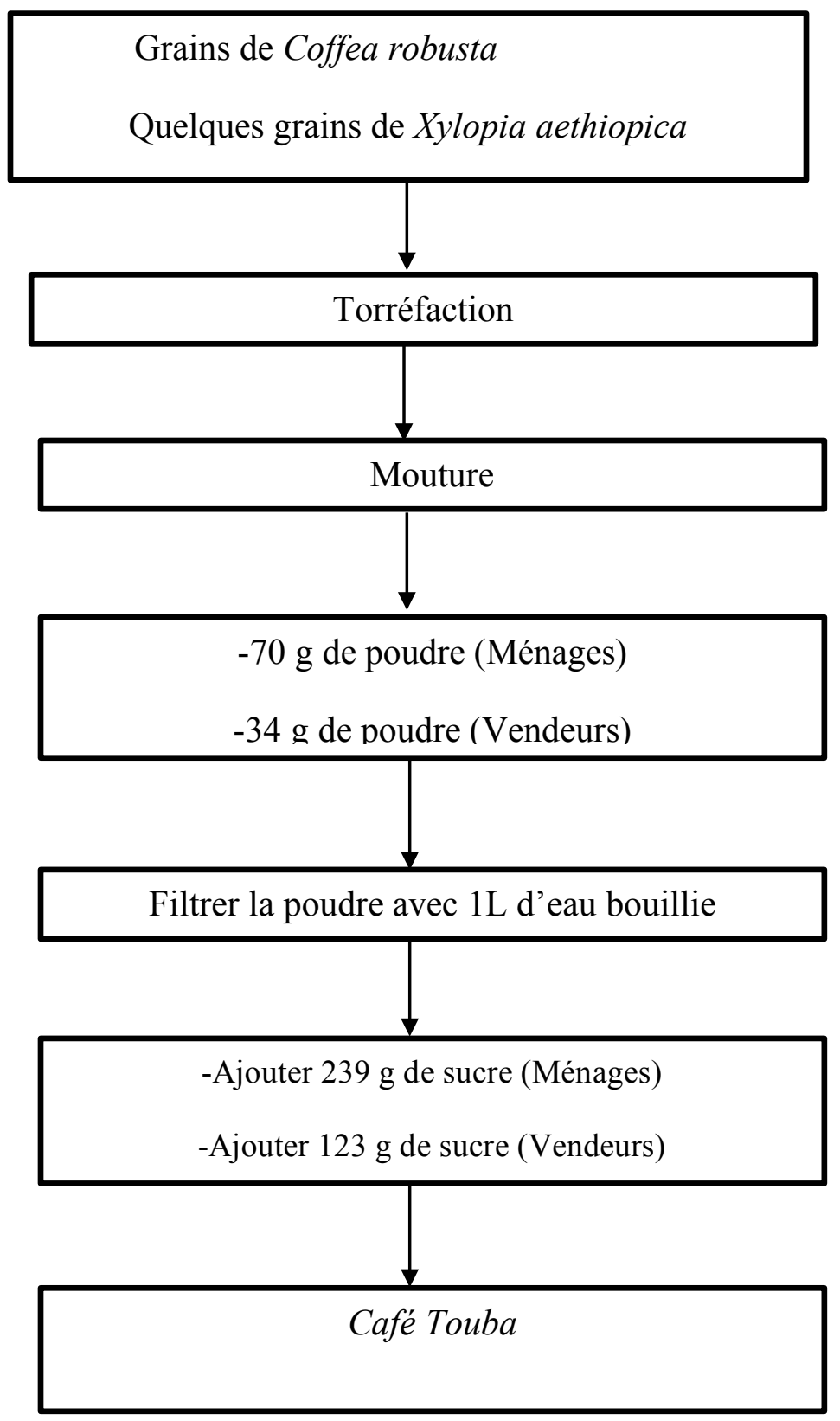

Figure 1 : Méthode de préparation du Café Touba 

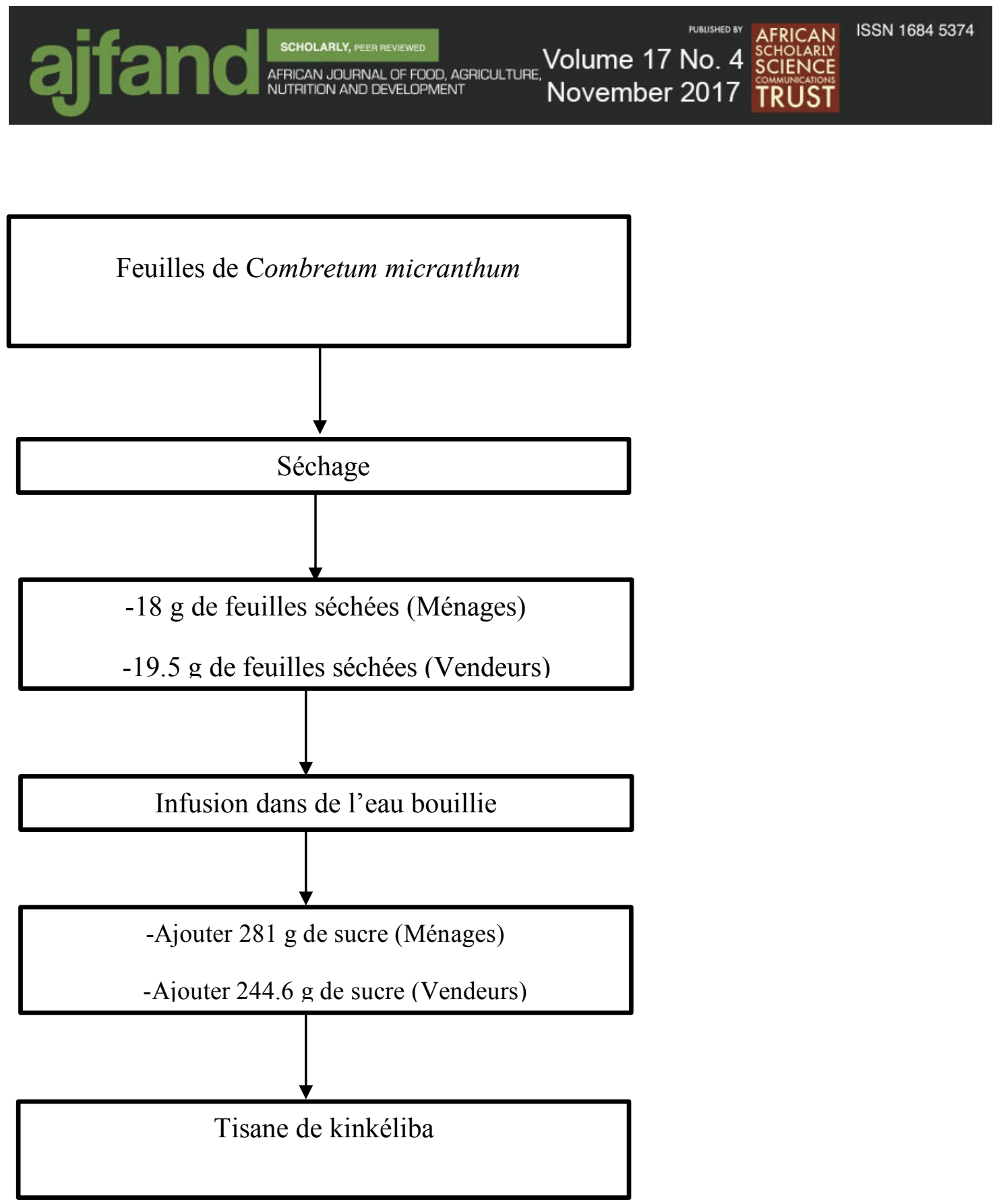

Figure 2 : Méthode de préparation de la tisane de kinkéliba

En se basant sur ces résultats de l'enquête, des méthodes standard ont été développées (Figure 1, Figure 2).

Les teneurs en tanins de ces boissons standardisées et la quantité de tanins ingérée au petit déjeuner à travers ces boissons par chaque client ou membre d'un ménage sont représentées dans le Tableau 5. Les résultats ont montré que la quantité de matière première utilisée pour la préparation des boissons et les volumes consommés déterminent les quantités de tanins ingérées. Le Café Touba préparé et consommé dans les ménages est la plus grande source de tanins parmi les boissons étudiées.

Ces boissons standards sont de sources de sucre. Dans les ménages, la concentration moyenne en sucre est de $239 \mathrm{~g} / \mathrm{L}$ pour le Café Touba et $281 \mathrm{~g} / \mathrm{L}$ pour la tisane de kinkéliba. 
Avec les volumes ingérés de Café Touba et de tisane de kinkéliba dans les ménages, les apports en sucre au petit déjeuner sont respectivement de 47,99g et 50,96g de sucre pour chaque membre des ménages. En ce qui concerne les vendeurs, la concentration moyenne en sucre est de $123 \mathrm{~g} / \mathrm{L}$ pour le Café Touba et $244,6 \mathrm{~g} / \mathrm{L}$ pour la tisane de kinkéliba. Chaque client qui consomme une tasse de tisane de kinkéliba ou de Café Touba ingère respectivement $53,39 \mathrm{~g}$ ou $17,03 \mathrm{~g}$ de sucre

\section{DISCUSSION}

Les modes de préparation de la tisane de kinkéliba sont relativement les mêmes aussi bien dans les ménages que chez les vendeurs. Cependant le volume de tisane de kinkéliba consommé au petit déjeuner dans les ménages $(181,36 \pm 66,62 \mathrm{ml})$ est nettement inférieur à celui acheté chez les vendeurs $(218,26 \pm 82,70 \mathrm{ml})$. Cette différence pourrait s'expliquer par le fait que les vendeurs de tisane de kinkéliba servent généralement dans des tasses plus volumineuses que celles utilisées dans les ménages. Contrairement à la tisane de kinkéliba, les vendeurs de Café Touba utilisent des tasses moins grandes que celles dans lesquelles le Café Touba est servi dans les ménages.

Le Café Touba préparé dans les ménages contient plus de poudre de café que celui qui est vendu. Les résultats ont montré des différences dans les modes de préparation du Café au sein des vendeurs. Ces différences peuvent être expliquées par des raisons économiques avec la recherche de plus de bénéfice de la part de certains vendeurs.

Les teneurs en tanins des boissons sont largement liées aux quantités de matières premières (poudre de Café Touba ou feuilles séchées de kinkéliba) utilisées lors de la préparation. Par conséquent, les ménages utilisant plus de poudre dans la préparation de Café Touba consomment plus de tanins provenant de ces boissons. Les tanins sont des polyphénols, qui jouent un rôle fonctionnel bénéfique pour la santé $[4,6]$. Ces substances phénoliques sont essentiellement des antioxydants qui contribuent au rôle protecteur que joue la tisane de kinkéliba et le café contre certaines maladies [1, 2,7, 8, 9]. Cependant, ces tanins peuvent jouer un rôle négatif dans l'absorption des minéraux tel que le fer [10]. Ainsi la consommation de la tisane de kinkéliba et du Café Touba pourrait affecter la biodisponibilité du fer augmentant ainsi les risques d'anémie ferriprive très fréquente dans les pays sous-développés comme le Sénégal [11]. Les tanins confèrent aux produits à base de substances végétales comme le Café Touba et la tisane de kinkéliba leur astringence $[12,13]$. A cause de cette astringence, du sucre est ajouté dans ces boissons pour améliorer leurs goûts. Par conséquent, ces boissons constituent ainsi des sources importantes de sucres pour les consommateurs. Les sucres naturellement présents dans certains aliments et les sucres ajoutés lors de la préparation des aliments constituent la somme des sucres ingérés. Ces sucres doivent apporter $45-65 \%$ des calories dans le régime normal d'une personne [14]. Selon les modes de consommation et les concentrations de sucre reportées dans cette étude, la tisane de kinkéliba préparée dans les ménages et par les vendeurs apportent respectivement 50,93g et 53,39g par tasse aux consommateurs. Pour le Café Touba, cette quantité est de 47,99g et $17.03 \mathrm{~g}$ pour les consommateurs des ménages et des clients, respectivement. Ainsi en appliquant les coefficients d'Atwater avec une valeur énergétique de $4 \mathrm{Kcal}$ pour 1 gramme de sucre, une seule prise de tisane de kinkéliba et de Café Touba apporterait respectivement 


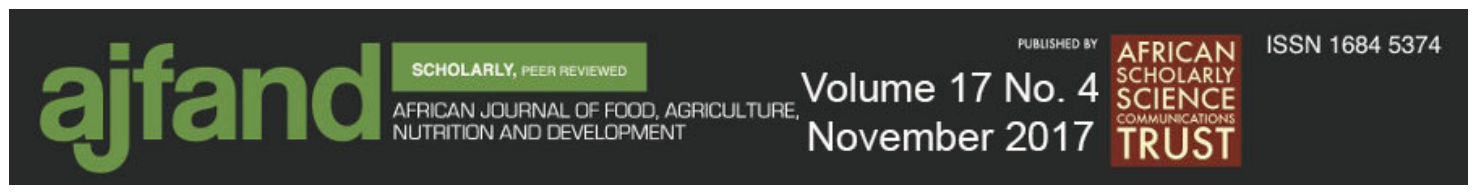

$203,84 \mathrm{Kcal}$ et $191,96 \mathrm{Kcal}$ à chaque consommateur au niveau des ménages. Chez les vendeurs, les apports énergétiques de tisane de kinkéliba et de Café Touba par tasse pour les clients sont respectivement de $213,56 \mathrm{Kcal}$ et $68,12 \mathrm{Kcal}$. Une seule prise de ces boissons apporte et peut même dépasser les quantités quotidiennes de sucres libres recommandées par l'Organisation Mondiale de la Santé (OMS). En effet, l'OMS recommande que les sucres ajoutés ne constituent pas plus de $10 \%$ des apports de calories quotidiens ce qui correspond à $50 \mathrm{~g}$ [15]. D'autres recommandations récentes fixent la quantité journalière de sucres ajoutée recommandée à $25 \mathrm{~g}$ pour prévenir des maladies chroniques [16].

\section{CONCLUSION}

La présente étude a permis de réaliser que les modes de préparation et de consommation de la tisane de kinkéliba et du Café Touba varient selon qu'on soit dans les ménages ou chez les vendeurs. Les quantités de sucres ajoutées pour corriger l'astringence de ces boissons pourraient causer des problèmes dans la mesure où ces doses permettent de dépasser celles recommandées par l'OMS augmentant ainsi les risques de survenue de maladies chroniques. 


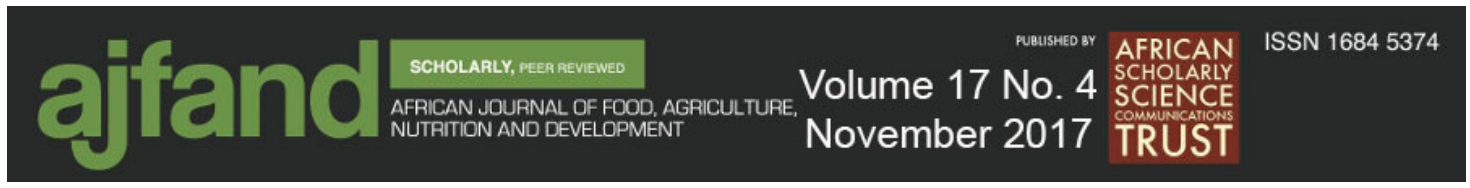

Tableau 1 : Mode de préparation et de consommation de la Tisane de kinkéliba

\begin{tabular}{|lllcc|}
\hline & Eau (L) & $\begin{array}{l}\text { Feuilles séchées } \\
\mathbf{( g )}\end{array}$ & Sucre $(\mathbf{g})$ & $\begin{array}{c}\text { Quantité } \\
\text { consommée/Personne } \\
(\mathbf{m l})\end{array}$ \\
\hline Ménages & $1,72 \pm 0,90$ & $31,2 \pm 19,78$ & $483,33 \pm 427,47$ & $181,36 \pm 66,62$ \\
\hline Vendeurs & $1,86 \pm 0.55$ & $36,46 \pm 22,12$ & $456,66 \pm 307,41$ & $218,26 \pm 82,70$ \\
\hline
\end{tabular}

Tableau 2: Mode de préparation et de consommation du Café Touba

\begin{tabular}{|lllll|}
\hline & Eau (L) & Poudre (g) & Sucre (g) & $\begin{array}{c}\text { Quantité } \\
\text { consommée/personne } \\
\text { (ml) }\end{array}$ \\
\hline Ménages & $1,53 \pm 0,54$ & $107,53 \pm 54,19$ & $366,66 \pm 99,85$ & $200,86 \pm 63,56$ \\
\hline Vendeurs & $17,56 \pm 44,33$ & $597 \pm 1255,52$ & $2158,33 \pm 3665,55$ & $138,53 \pm 37,02$ \\
\hline
\end{tabular}

Tableau 3: Mode de préparation standardisé à $1 \mathrm{~L}$ de Café Touba

\begin{tabular}{|lcc|}
\hline & Ménages & Vendeurs \\
\hline Quantité eau (L) & 1 & 1 \\
\hline Quantité poudre (g) & 70 & 34 \\
\hline Quantité sucre (g) & 239 & 123 \\
\hline
\end{tabular}




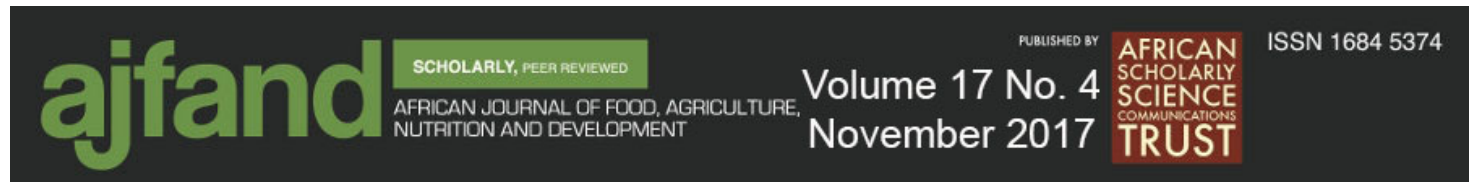

Tableau 4: Mode de préparation standardisé à $1 \mathrm{~L}$ de Tisane de kinkéliba

\begin{tabular}{|lcc|}
\hline & Ménages & Vendeurs \\
\hline Quantité eau (L) & 1 & 1 \\
\hline Quantité feuilles séchées (g) & 18 & 19.5 \\
\hline Quantité sucre (g) & 281 & 244.6 \\
\hline
\end{tabular}

Tableau 5: Volumes de boisson et quantités de tanins ingérées par une seule prise

\begin{tabular}{|l|c|c|c|c|}
\cline { 2 - 5 } \multicolumn{1}{c|}{} & \multicolumn{2}{c|}{ Ménages } & \multicolumn{2}{c|}{ Vendeurs } \\
\cline { 2 - 5 } \multicolumn{1}{c|}{$\begin{array}{l}\text { Café } \\
\text { Touba }\end{array}$} & $\begin{array}{l}\text { Tisane de } \\
\text { Kinkéliba }\end{array}$ & $\begin{array}{l}\text { Café } \\
\text { Touba }\end{array}$ & $\begin{array}{c}\text { Tisane } \\
\text { de Kinkéliba }\end{array}$ \\
\hline Tanins (g/L) & 3,2 & 2 & 1,2 & 2 \\
\hline Volume ingéré par tasse (ml) & 200,8 & 181,36 & 138,5 & 218,26 \\
\hline Quantité de tanins ingérée par tasse $(\mathrm{g})$ & 0,64 & 0,36 & 0,17 & 0,44 \\
\hline
\end{tabular}




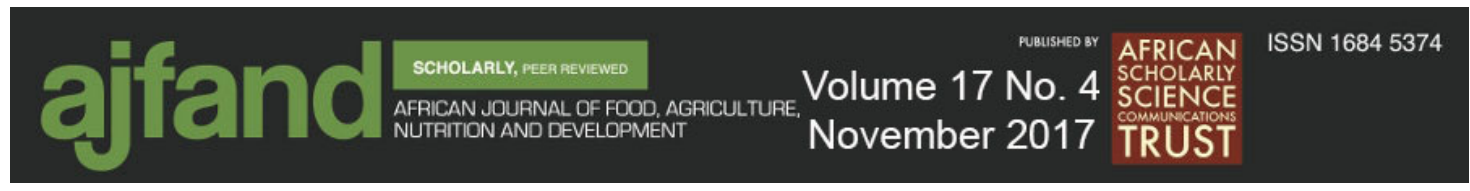

\section{BIBLIOGRAPHIE}

1. Bidel S, Hu G,Qiao Q,Jousilahti P, Antikainen $\mathbf{R}$ and $\mathbf{J}$ Tuomilehto Coffee consumption and risk of total and cardiovascular mortality among patients with type 2 diabetes. Diabetologia, 2006; 49: 2618-2626.

2. Touré $\mathbf{A}, \mathbf{X u} \mathbf{X}$, Michel $\mathbf{T}$ and $\mathbf{M}$ Bangoura In vitro antioxidant and radical scavenging of Guinean kinkeliba leaf (Combretum micranthum G. Don) extracts. Nat Prod Res. 2011; 11: 1025-36.

3. Alonso-Díaz MA, Torres-Acosta JFJ, Sandoval-Castro CA and H Hoste Tannins in tropical tree fodders fed to small ruminants: A friendly foe? Small Ruminant. Research, 2010; 89: 164-173.

4. Chung KT, Wong TY, Wei CI, Huang YW and Y Lin Tannins and human health: a review. Crit. Rev. Food Sci. Nutr. 1998; 38(6): 421-64.

5. Seigler DS, Seilheimer S, Keesy $\mathbf{J}$ and HF Huamg Tanins from four common acacia species of Texasand Northeastern Mexico. Econ. Bot. 1986; 40: 220-232.

6. Belobrajdic DP and AR Bird The potential role of phytochemicals in wholegrain cereals for the prevention of type-2 diabetes. Nutrition Journal, 2013 ; 12: 62

7. Stevenson DE and RD Hurst Polyphenolic phytochemicals - just antioxidants or much more? Cell. Molec. Life Sci., 2007; 64(22): 2900-2916.

8. Liu H, Hu GH, Wang XC, Huang TB, Xu L, Lai P, Guo ZF and YF Xu Coffee Consumption and Prostate Cancer Risk: A Meta-Analysis of Cohort Studies. Nutr. Cancer. 2015; 1-9.

9. Rosendahl AH, Perks CM, Zeng L, Markkula A, Simonsson M, Rose C, Ingvar C, Holly JM and H Jernström Caffeine and caffeic acid inhibit growth and modify estrogen receptor (ER) and insulin-like growth factor I receptor (IGFIR) levels in human breast cancer. Clin. Cancer Res. 2015.

10. Sotelo A, González-Osnaya L, Sánchez-Chinchillas A and A Trejo Role of oxate, phytate, tannins and cooking on iron bioavailability from foods commonly consumed in Mexico. Int. J. Food Sci. Nutr. 2010; 61(1): 29-39.

11. Scott SP, Chen-Edinboro LP, Caulfield LE and LE Murray-Kolb The impact of anemia on child mortality: an updated review. Nutrients, 2014; 6(12): 59155932.

12. Sarni-Manchado $\mathbf{P}$ and $\mathbf{V}$ Cheynier Les polyphénols en agroalimentaire, Lavoisier, Editions Tec \& Doc, 2006: 398. 


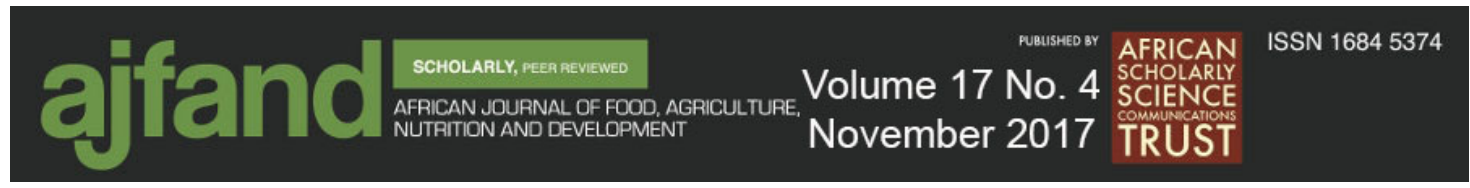

13. Sheridan MK and RJ Elias Exogenous acetaldehyde as a tool for modulating wine color and astringency during fermentation. Food Chem. 2015; 177: 17-22.

14. U.S. Department of Agriculture Dietary Guidelines for Americans.2010.

15. OMS. Organisation mondiale de la santé: Diet, nutrition and the prevention of chronic diseases: report of a joint WHO/FAO expert consultation. 2003: 54-70.

16. Jennifer JO, Jennifer PH and L D Meyers Dietary Reference Intakes The Essential Guide to Nutrient Requirements. The national academies press Washington, D.C. 2006. 